\title{
Macroporous silica-alumina composites with mesoporous walls
}

\author{
GAUTAM GUNDIAH \\ Chemistry and Physics of Materials Unit, Jawaharlal Nehru Centre for Advanced Scientific Research, Jakkur P.O., \\ Bangalore 560 064, India
}

MS received 16 February 2001

\begin{abstract}
Macroporous silica-alumina composites with mesopores have been prepared by employing polymethylmethacrylate beads as templates in the presence of the cationic surfactant, N-cetyl-N,N,Ntrimethylammonium bromide. The $\mathrm{Si} / \mathrm{Al}$ ratio in the composites has been varied between 4.5 and 48 and the occurrence of mesopores has been verified by $\mathrm{X}$-ray diffraction. The surface areas of the samples vary between 676 and $1038 \mathrm{~m}^{2} \mathrm{~g}^{-1}$, with the highest value in the sample with $\mathrm{Si} / \mathrm{Al}=48$.
\end{abstract}

Keywords. Macroporous materials; mesoporous materials; silica-alumina composites.

\section{Introduction}

Macroporous materials of oxides such as $\mathrm{TiO}_{2}, \mathrm{SiO}_{2}$ (Holland et al 1998, 1999) and ferroelectric $\mathrm{PbTiO}_{3}$ (Gundiah and Rao 2000) are attracting much attention. Macroporous solids with micropores or mesopores would make useful adsorbents or catalyst supports. Recently, Stein and coworkers have reported the synthesis of macroporous silica containing mesoporous walls by treating a solution containing the silica precursor with an aqueous mixture of cetyltrimethylammonium hydroxide and tetrapropylammonium hydroxide and pouring the solution over an ordered array of polystyrene beads (Holland et al 1999). We have attempted to make macroporous aluminosilicates containing mesoporous walls. For this purpose we have employed an ordered array of polymethylmethacrylate spheres in the presence of the cationic surfactant N-cetyl-N,N,N-trimethylammonium bromide (CTAB). We describe the macroporous-mesoporous silicaalumina composites with satisfactory/high surface areas.

\section{Experimental}

Polymethylmethacrylate (PMMA) spheres of diameter $275 \mathrm{~nm}$ were obtained from Soken Chemicals, Japan. These were taken as $1 \% \mathrm{wt}$ : wt in distilled water and sonicated for $30 \mathrm{~min}$ to disperse the spheres. This sol was centrifuged at $1000 \mathrm{rpm}$ for $6-8 \mathrm{~h}$. The water above the spheres was removed with a pipette carefully and the spheres were left to dry under ambient conditions. These formed millimeter sized colloidal crystals which were used for further reactions.

The silica-alumina gel was synthesized in the following manner. To a solution of $0.5 \mathrm{~g}$ of CTAB in $10 \mathrm{ml} \mathrm{H}_{2} \mathrm{O}$, $0 \cdot 1 \mathrm{~g}$ of $\mathrm{Al}_{2}\left(\mathrm{SO}_{4}\right)_{3} \cdot 16 \mathrm{H}_{2} \mathrm{O}$ was added and stirred until a homogenous solution was obtained. $1 \mathrm{ml}$ of tetraethylorthosilicate was added to this mixture and the stirring continued. Finally, a few drops of $\mathrm{NH}_{3}$ (25\% solution) were added to make the $\mathrm{pH} \sim 8$. The turbid solution was poured on the colloidal crystals to allow it to percolate through the voids between the spheres. This was left to dry at room temperature. The dried products were calcined at $400^{\circ} \mathrm{C}$ for $2 \mathrm{~h}$, which resulted in sample $\mathrm{A}$ shown in table 1 . By a similar procedure, we prepared two other silica-alumina samples (B and C) with different $\mathrm{Si} / \mathrm{Al}$ ratios.

Powder X-ray diffraction (XRD) patterns of the samples were obtained using $\mathrm{CuK} \alpha$ radiation (Rich-Siefert, XRD3000-TT). Thermogravimetric analysis (TGA) was carried out (Mettler Toledo, TG-850) in flowing oxygen atmosphere $\left(50 \mathrm{ml} \cdot \mathrm{min}^{-1}\right)$ at a heating rate of $10 \mathrm{~K} \mathrm{~min}^{-1}$. Scanning electron microscopy (SEM) and energy-dispersive X-ray (EDX) analysis were carried out using a Leica scanning electron microscope fitted with EDX spectrometer and a Link ISIS detector. Nitrogen adsorption studies were carried out at liquid nitrogen temperature on these samples using a Cahn-2000 Electrobalance. Solid state MAS NMR measurements were performed on a Bruker DSX-300 MHz Spectrometer. Tetramethylsilane (TMS) and $\mathrm{Al}\left(\mathrm{H}_{2} \mathrm{O}\right)_{6}^{3+}$ were used as external standards.

\section{Results and discussion}

Table 1 lists the compositions of the various samples prepared by us. In figure 1a we show the SEM image of the templating PMMA spheres employed by us. The spheres are of uniform diameter and are ordered into arrays. In figure $1 \mathrm{~b}$ we show the SEM image of sample B after the spheres were treated with the silica-alumina gel, dried and calcined. The networks are fairly well ordered. 
The diameter of the pores is slightly smaller $(\sim 150 \mathrm{~nm})$ than that of the initial PMMA spheres. The shrinkage occurs during the removal of the template during calcination. The SEM image of the samples A and C too were similar to the image in figure $1 \mathrm{~b}$. There was no noticeable change in the external morphology on increasing the amount of aluminum in the samples. The SEM images clearly establish the presence of macropores probably connected in three dimensions.

XRD patterns of the as-synthesized and calcined samples are presented in figure 2. The as-synthesized

Table 1. Properties of macroporous $\mathrm{SiO}_{2}-\mathrm{Al}_{2} \mathrm{O}_{3}$ with mesoporous walls.

\begin{tabular}{|c|c|c|c|c|c|}
\hline & \multicolumn{2}{|c|}{$\mathrm{Si} / \mathrm{Al}$} & \multicolumn{2}{|c|}{$d(\AA)$} & \multirow{2}{*}{$\begin{array}{l}\text { Surface area } \\
\qquad\left(\mathrm{m}^{2} \mathrm{~g}^{-1}\right)\end{array}$} \\
\hline & Gel & Product & As-synth. & Calcined & \\
\hline A & $18 \cdot 5$ & $48 \cdot 0$ & 38.94 & $35 \cdot 60$ & 1038 \\
\hline B & $12 \cdot 4$ & $15 \cdot 3$ & $35 \cdot 26$ & 32.64 & 804 \\
\hline $\mathrm{C}$ & $9 \cdot 3$ & $4 \cdot 5$ & $38 \cdot 15$ & $33 \cdot 52$ & 676 \\
\hline
\end{tabular}

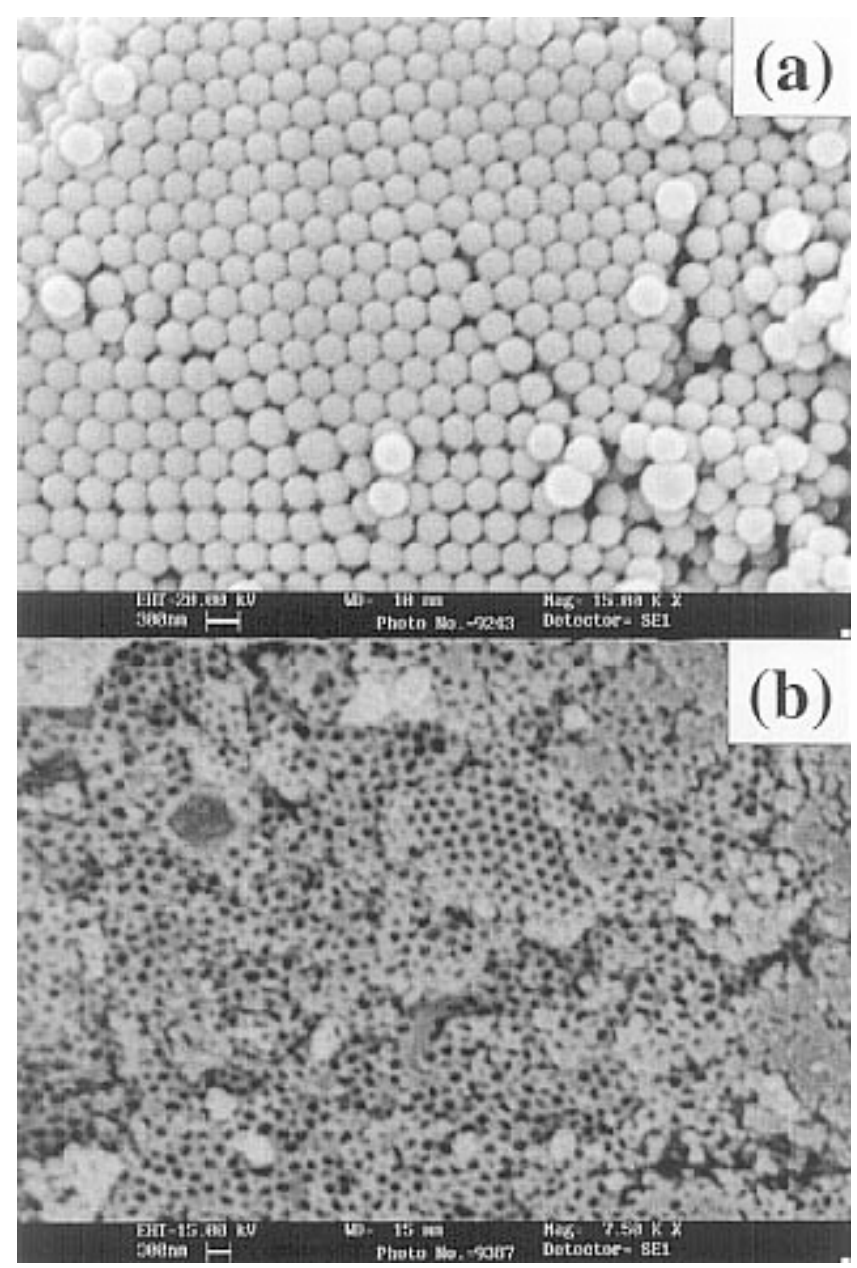

Figure 1. a. SEM image of PMMA spheres of $275 \mathrm{~nm}$ diameter and b. SEM image of sample B after removal of the templates. samples of $\mathrm{A}, \mathrm{B}$ and $\mathrm{C}$ show single peaks with $d$-values $38.94,35.26$ and $38.15 \AA$ respectively. The low-angle peak is similar to that shown by hexagonal mesoporous solids. There is a decrease in the $d$-value after calcination due to removal of template CTAB molecules, $d$-values being $35.5,32.64$ and $33.52 \AA$ respectively for A, B and C. The presence of the low angle peak after calcination indicates that the mesoporous phase is reasonably intact after removal of the template. We know of three materials, $\mathrm{A}, \mathrm{B}$, and $\mathrm{C}$, which are macroporous-mesoporous.

TGA curves of the aluminosilicates $A$ and $C$ are shown in figure 3 . The as-synthesized samples show a single weight loss from $250-350^{\circ} \mathrm{C}$ due to the loss of the PMMA and CTAB templates. The weight loss above $350^{\circ} \mathrm{C}$ is due to the condensation of the silanol that occurs after $350^{\circ} \mathrm{C}$. The TGA curve for the calcined samples show a single weight loss corresponding to the adsorbed water. The amount of template removed was calculated to be above $95 \%$.

We performed MAS NMR studies to determine the coordination of aluminium and silicon in the framework. ${ }^{29} \mathrm{Si}$ MAS NMR of the as-synthesized sample A showed two broad peaks at -101 and $-110 \mathrm{ppm}$ corresponding to the $Q^{3}$ and $Q^{4}$ species respectively. The intensity of the $Q^{3}$ species was greater than the $Q^{4}$ species indicating that the silanol groups had not condensed fully. On calcination, the relative intensity of the $Q^{4}$ species increased, showing
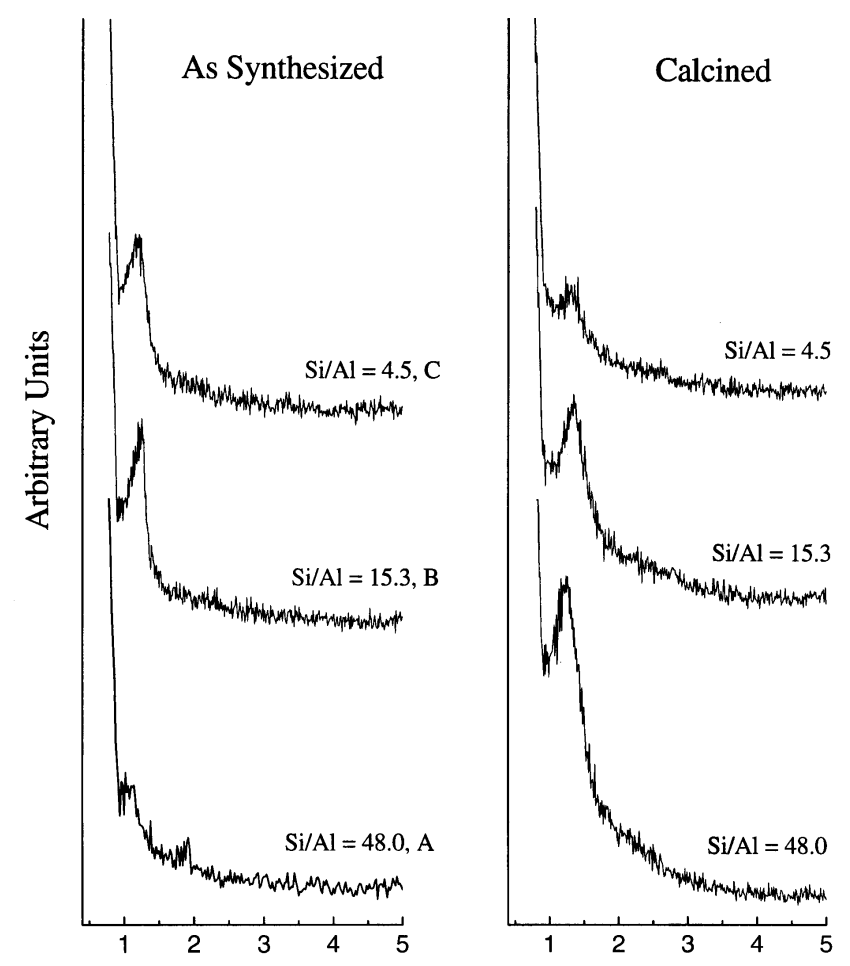

Theta (degrees)

Figure 2. XRD patterns of the as-synthesized and calcined samples A, B and C. 

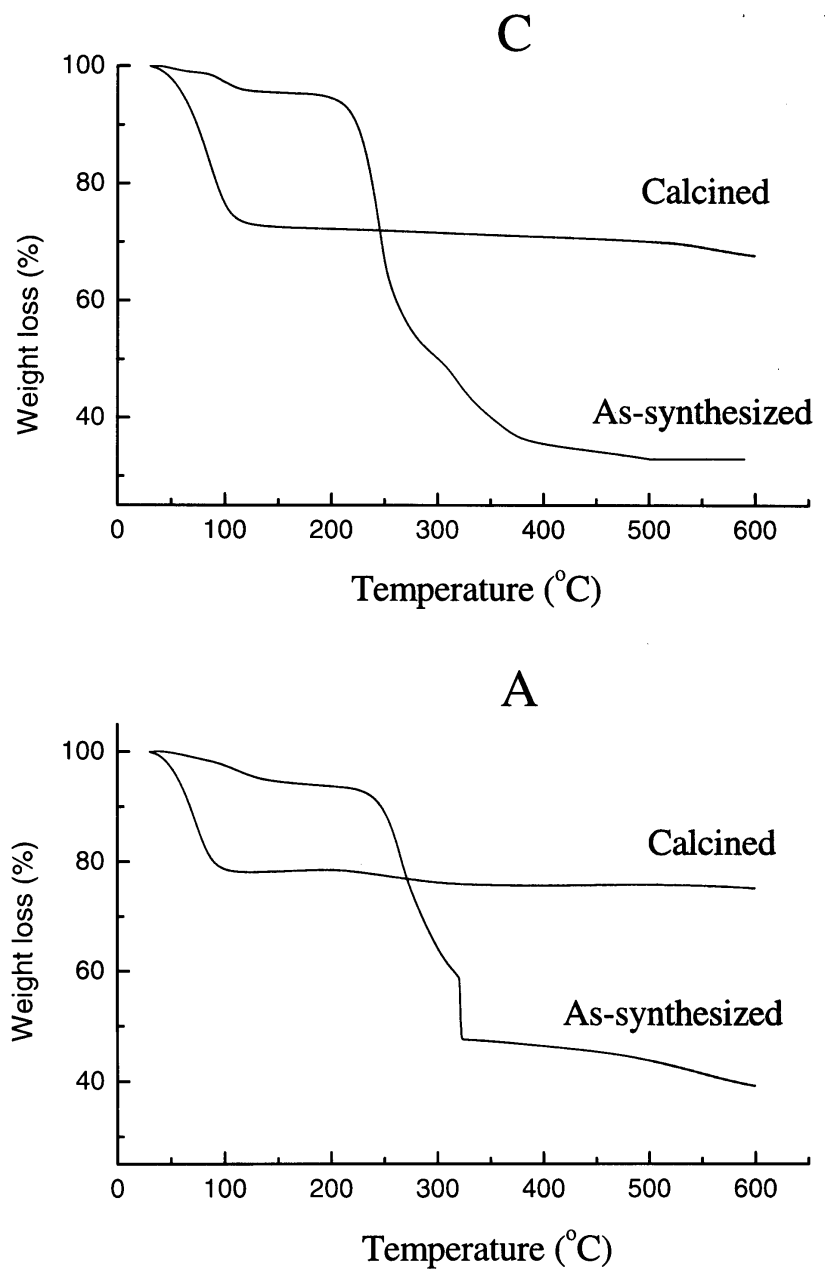

Figure 3. TGA curves of the aluminosilicate samples A and C.

that on calcination the silanol groups condense to form Si-O-Si species. ${ }^{27} \mathrm{Al}$ MAS NMR of the calcined sample A showed a large narrow peak at -7 ppm corresponding to octahedrally coordinated aluminium species and a very small, broad feature centred at $46 \mathrm{ppm}$ due to tetrahedrally coordinated aluminium. On integrating these peaks, the areas under the peaks were found to be in the ratio of $1: 0 \cdot 2$ of the octahedral to the tetrahedral species. It appears only $20 \%$ of the $\mathrm{Al}$ is present in the framework. Most of the $\mathrm{Al}$ is therefore present as $\mathrm{Al}_{2} \mathrm{O}_{3}$ in composite form with $\mathrm{SiO}_{2}$. This is because of the limitation due to the $\mathrm{pH}$ and temperature employed in the preparation. We may therefore consider $\mathrm{A}, \mathrm{B}$, and $\mathrm{C}$ as composites of $\mathrm{SiO}_{2}$ and $\mathrm{Al}_{2} \mathrm{O}_{3}$ with a small proportion of aluminosilicate. Even though the amount of aluminium in the framework is small, the composite materials that we have made have macroporous-mesoporous characteristics described earlier.

Nitrogen adsorption isotherms of the samples A, B and $\mathrm{C}$ are presented in figure 4 . These are typical type IV isotherms characteristic of mesoporous materials. The isotherms exhibit little or no hysteresis, showing thereby that the pores may not be very ordered. The surface areas
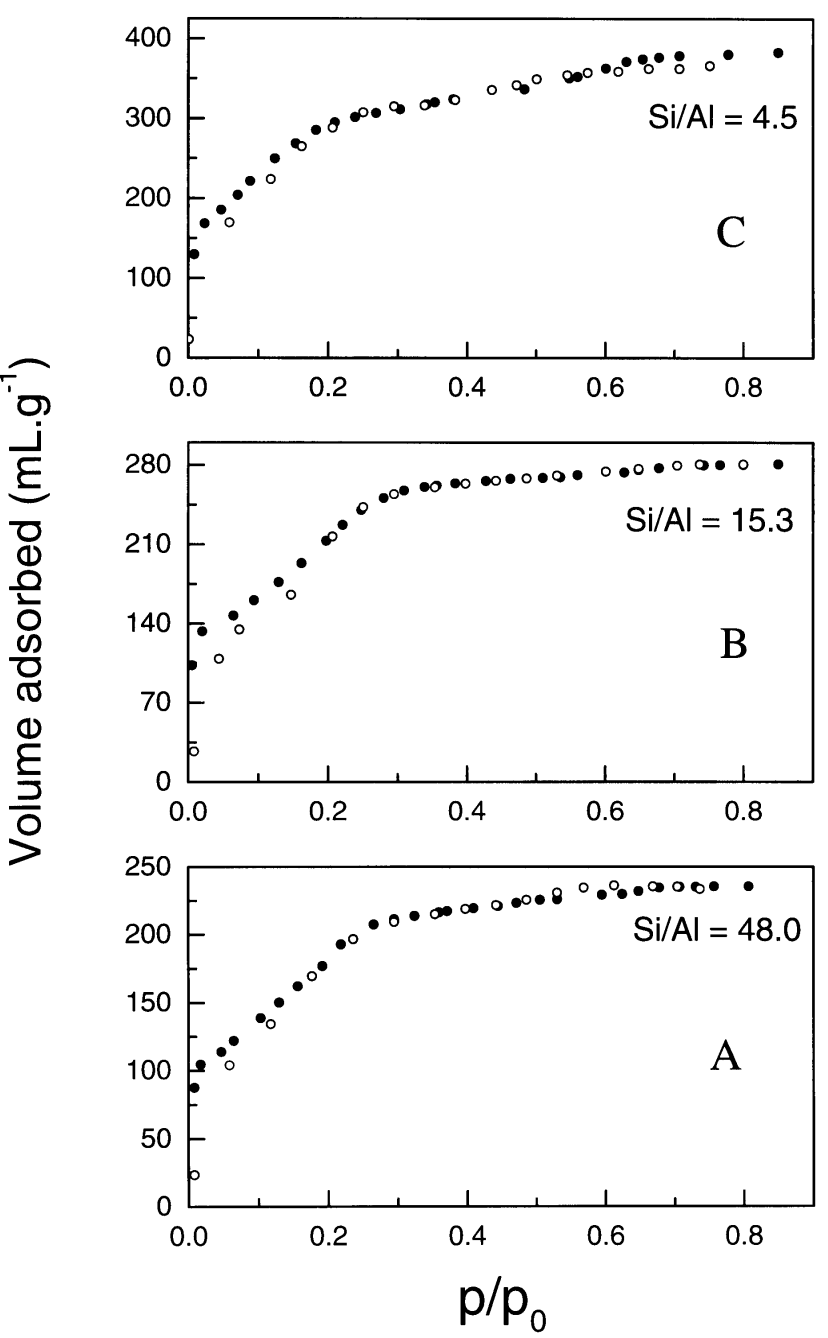

Figure 4. Nitrogen adsorption isotherms for samples A, B and C. The dark circles represent the adsorption cycle and the open circles represent the desorption cycle.

calculated by the BET method are in the range of 676$1038 \mathrm{~m}^{2} \mathrm{~g}^{-1}$ for the three samples. There is a decrease in the surface area with the increasing aluminum content, consistent with the observations of Janicke et al (1999), who observed that the surface areas decrease as we decrease the $\mathrm{Si} / \mathrm{Al}$ ratios.

\section{Conclusions}

We have demonstrated the synthesis of macroporous aluminosilicates containing mesoporous walls. Though the amount of aluminium in the framework is small, the presence of macropores as well as mesopores makes these composites suitable as catalyst supports. The presence of macropores would aid in the diffusion of species to the active sites of the catalyst. The study opens up scope for preparing macroporous-mesoporous structures by doping other transition metals such as $\mathrm{Ti}, \mathrm{V}, \mathrm{Cr}$, etc into the silica framework. The only limiting factor is that the reaction 
has to be carried out at a temperature below which the template decomposes.

\section{Acknowledgements}

The author thanks Prof. C N R Rao for suggesting the problem and his guidance during the course of its investigation. The author also thanks SIF, IISc for the MAS NMR measurements.

\section{References}

Gundiah G and Rao C N R 2000 Solid State Sci. 2877

Holland B T, Blanford C F and Stein A 1998 Science 281 538

Holland B T, Blanford C F, Do T and Stein A 1999 Chem. Mater. 11798

Janicke M T, Landry C C, Christiansen S C, Birtalan S, Stucky G D and Chmelka B F 1999 Chem. Mater. 111342 